\title{
Comparative genomic hybridization analysis of thymic neuroendocrine tumors
}

\author{
Chin-Chen Pan ${ }^{1,2}$, Yiin-Jeng Jong ${ }^{3}$ and Yann-Jang Chen ${ }^{4,5}$ \\ ${ }^{1}$ Department of Pathology, National Yang-Ming University, Taipei, Taiwan; ${ }^{2}$ Taipei Veterans General \\ Hospital, Taiwan; ${ }^{3}$ Institute of Genetics, National Yang-Ming University, Taipei, Taiwan; ${ }^{4}$ Faculty of Life \\ Sciences, National Yang-Ming University, Taipei, Taiwan and ${ }^{5}$ Department of Pediatrics, Taipei Veterans \\ General Hospital, Taiwan
}

\begin{abstract}
Thymic neuroendocrine (carcinoid) tumors are a rare neoplasm of the anterior mediastinum. The tumors frequently exhibit a wide spectrum of histology and appear to follow a more aggressive behavior than their nonthymic counterparts. Given the differing clinicopathologic manifestations, thymic neuroendocrine tumors may also possess different cytogenetic abnormalities from those that occur in foregut carcinoid tumors. In this study, we employed comparative genomic hybridization to detect genomic instability in 10 sporadic thymic neuroendocrine tumors and one multiple endocrine neoplasia type 1 (MEN1)-associated case. Gross chromosomal imbalances were found in nine cases, including gains of chromosomal material on regions $\mathbf{X}$, 8, 18 and 20p and losses on 3, 6, 9q, 13q and 11q. We did not observe deletion at locus 11q13 where the MEN1 gene is located. These findings were essentially dissimilar to those reported in sporadic and MEN1-associated foregut carcinoid tumors. Consequently, we consider that a distinctive cytogenetic mechanism is at work in the development of thymic neuroendocrine tumors, which is different from that of foregut carcinoid tumors.

Modern Pathology (2005) 18, 358-364, advance online publication, 23 July 2004; doi:10.1038/modpathol.3800246
\end{abstract}

Keywords: thymic neuroendocrine tumor; carcinoid; CGH; MEN1

The term thymic carcinoid tumor was first introduced by Rosai and Higa ${ }^{1}$ to denote a group of thymic neoplasms that histologically resemble the carcinoid tumors of other systems. Later series of studies demonstrated that this group of neoplasms frequently displays a diversity of morphologic features ranging from typical carcinoid as those seen in the bronchopulmonary and digestive tracts, to poorly differentiated small-cell carcinoma. In addition, many such tumors behave more aggressively than the conventional nonthymic carcinoid tumors. Reportedly around half of the cases invaded the surrounding mediastinal structures, and $30-40 \%$ metastasized. ${ }^{2-4}$ Consequently, Moran and Suster ${ }^{3}$ proposed to designate the entity as thymic neuroendocrine carcinoma with a threetiered (low, intermediate and high) grading system, which is roughly equivalent to the classic carcinoid, atypical carcinoid and small-cell undifferen-

Correspondence: Dr Yann-Jang Chen, MD, PhD, Faculty of Life Sciences, National Yang-Ming University, No. 155, Li-Nong Street, Sec. 2, Taipei 11217, Taiwan.

E-mail: ccpan@vghtpe.gov.tw

Received 24 May 2004; revised and accepted 28 June 2004; published online 23 July 2004 tiated carcinoma of the World Health Organization (WHO) classification. ${ }^{5}$

Owing to differing pathologic and clinical presentation, we postulate a different cytogenetic oncogenesis between the thymic and foregut neuroendocrine tumors. Nevertheless, few cytogenetic studies specifically addressing the thymic neuroendocrine tumors are available. So far, only a total of 12 cases in three reports of multiple endocrine neoplasia type 1 syndrome (MEN1)-associated thymic neuroendocrine tumors were examined by using loss of heterozygosity (LOH) study at the $11 \mathrm{q} 13$ locus where MEN1 gene is located. ${ }^{6-8}$ The cytogenetic and molecular events underlying the development of thymic neuroendocrine tumor remain largely unknown. In this study, we aim to characterize the chromosomal aberrances in thymic neuroendocrine tumors with comparative genomic hybridization (CGH) analysis and compare our findings with those of previous reports.

\section{Materials and methods}

Case Selection

We retrieved 11 cases of thymic neuroendocrine tumors from the surgical pathology archive at Taipei 
Veterans General Hospital from 1985 to 2000. The specimens were obtained through autopsy, radical surgery, tumor removal and debulking. Small biopsies unsuitable for further evaluation were excluded. These cases had complete clinicopathologic evaluations, including image studies to ascertain the mediastinal primary and absence of pulmonary lesions, and to rule out metastatic diseases.

The hematoxylin and eosin slides of each case were reviewed and subtyped as low-grade, intermediate-grade and high-grade neuroendocrine tumors (Figure 1) based on the criteria proposed by Moran and Suster. ${ }^{3}$ Briefly, the low-grade (welldifferentiated) tumor is composed of uniform cells in prominent organoid pattern with mild atypism and rare necrosis and mitoses. The intermediategrade (moderately differentiated) tumor displays increased mitotic activity, solid growth and necrosis. The high-grade (poorly differentiated) tumor is characterized by solid infiltrative masses with marked atypism, extensive necrosis and high mitotic count. Immunohistochemical stains for epithelial markers and neuroendocrine markers (eg chromogranin A, synaptophysin, CD56) were performed to support the diagnosis.

The tumors were staged according to the WHO classification of thymic tumors ${ }^{5}$ as Stage I, encapsulated; stage II, minimally invasive, that is, surrounded by a capsule which is microscopically penetrated by tumor growth or which invades the mediastinal fat; stage III, widely invasive, that is, spreading by direct extension into adjacent structures such as pericardium, large vessels, or lung; and stage IV, with implants or metastases.

\section{DNA Extraction}

Since no frozen specimens were available for this rare tumor, we adopted a highly effective method to extract DNA from formalin-fixed, paraffin-embedded samples as previously described. ${ }^{9}$ Briefly, portions of the tumors from the specimens were identified under the microscope by a pathologist (CC Pan) and were then cut into small pieces to include as little paraffin as possible, then incubated in xylene at $45^{\circ} \mathrm{C}$ for $1 \mathrm{~h}$, and washed twice with $70 \%$ ethanol. After complete drying in air, $1 \mathrm{ml}$ of lysis buffer (100 mM Tris-HCl, $\mathrm{pH} 7.8 ; 5 \mathrm{mM}$ ethylenediaminetetraacetic acid; $0.2 \%$ sodium dodecyl sulfate and $200 \mathrm{mM} \mathrm{NaCl}$ ) supplemented with proteinase $\mathrm{K}$ at a final concentration of $0.3 \mathrm{mg} / \mathrm{ml}$ was added and incubated at $55^{\circ} \mathrm{C}$ for $72 \mathrm{~h}$; additional proteinase $\mathrm{K}(10 \mathrm{ml}$ of $20 \mathrm{mg} / \mathrm{ml}$ stock solution) was added at 24 and $48 \mathrm{~h}$. DNA was then extracted by using the standard phenolchloroform-isoamyl alcohol method. DNA samples were quantified using a DyNA Quant 200 fluorometer (Hoefer, Pharmacia Biotech, San Francisco, CA, USA).
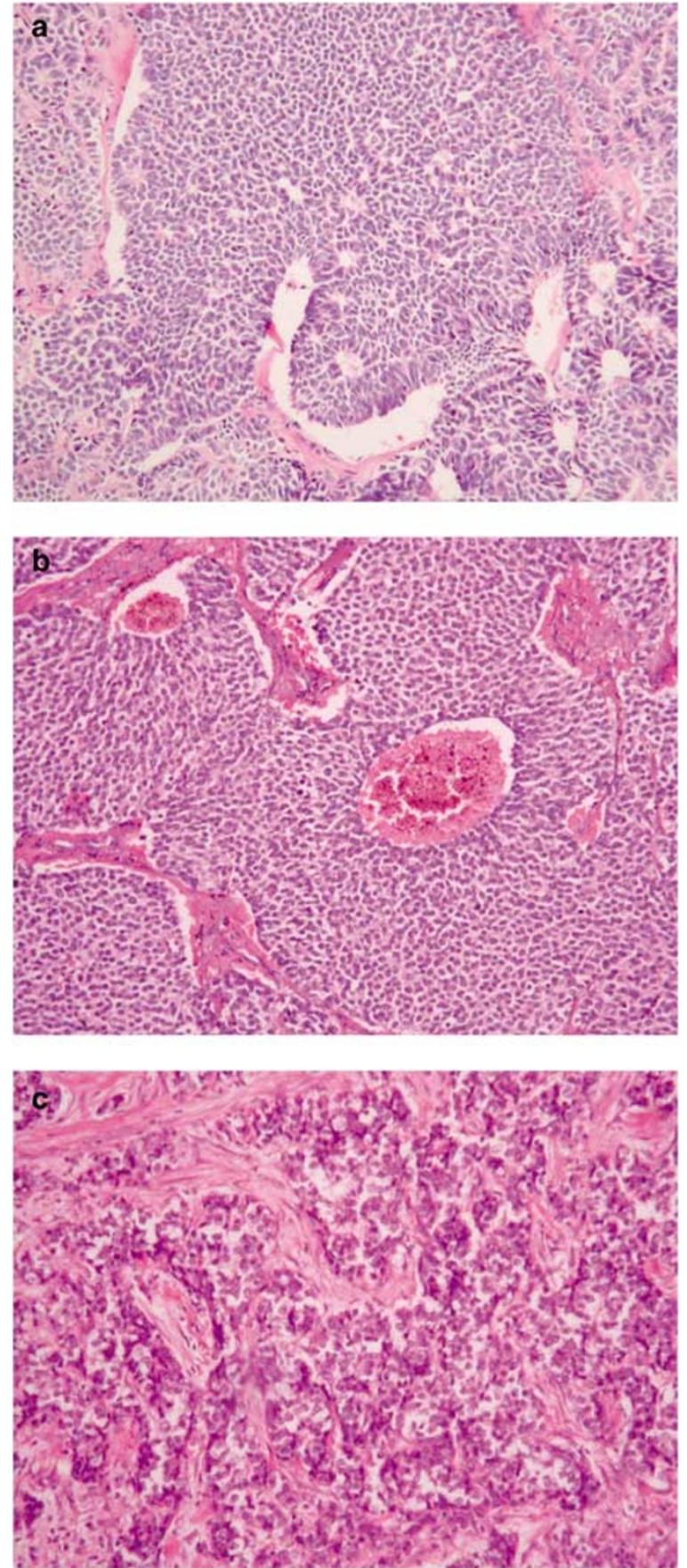

Figure 1 Histology of thymic neuroendocrine (carcinoid) tumor. (a) Low-grade tumor shows uniform cells in organoid pattern without necrosis. (b) Intermediate-grade tumor reveals more solid areas and punctate necroses. (c) High-grade tumor consists of infiltrative masses of small anaplastic cells.

\section{CGH}

Amounts of 500 ng of DNA extracted from the tumor samples or normal placenta were each labeled with fluorescein-conjugated 12-dUTP or Spectrum Redconjugated dUTP by nick translation. The sizes of the resulting probes ranged typically from 500 to 2000 base pairs. Slides containing metaphase 
chromosomes were prepared using blood from volunteers. Peripheral blood lymphocytes were cultured and stimulated with phytohemagglutinin according to standard procedures. For DNA hybridization, the metaphase chromosome templates were denatured at $76^{\circ} \mathrm{C}$ for $3 \mathrm{~min}$ in $70 \%$ deionized formamide and $2 \times$ SSC $(0.3 \mathrm{M} \mathrm{NaCl}, 0.03 \mathrm{M}$ sodium citrate, $\mathrm{pH}$ 7.0), then dehydrated by gradient ethanol and air-dried. Amounts of $200 \mathrm{ng}$ of the labeled probes from both tumor and control samples were then ethanol-precipitated with $10 \mu \mathrm{g}$ of human Cot-1 DNA and dissolved in $10 \mu$ l hybridization solution (Master Mix 1.0: 70\% formamide, 14.3\% dextran sulfate and $2.8 \times \mathrm{SSC}$ ) to form the probe mixture. The mixed probes were heat-denatured at $76^{\circ} \mathrm{C}$ for $7 \mathrm{~min}$ and competitively hybridized to the metaphase chromosome templates at $37^{\circ} \mathrm{C}$ for $36-72 \mathrm{~h}$ in a humid chamber. After hybridization, the slides were washed and counterstained with $0.1 \mathrm{mg} / \mathrm{ml}$ 4,6-diamidino-2-phenylindole (DAPI).

The CGH hybridization patterns were then typically analyzed using QUIPS XL genetic workstation (Vysis, Downeers Grove, IL, USA) that included programs for both image acquisition and analysis. The fluorescent images were first observed under a microscope equipped with standard fluorescence system (Axioscope, Carl Zeiss, Germany) and a cooled-CCD camera (Photometrics, Tucson, AZ, USA). Images from at least 6-10 complete assemblies of metaphase chromosome were captured using the SmartCapture software of the QUIPS program. To facilitate sequential observation and perfect registration of images from three different fluorescence channels, all excitation filters were mounted and controlled by a Ludl filter wheel (Ludl Electronic Products, Hawthrone, NY, USA). Also, a triple-dichroid mirror and a triple-bandpass emission filter (Chroma Technology, Brattleboro, VT, USA) were used in the microscope to avoid manual switching when changing fluorescence channels. The blue fluorescence images of DAPI-stained chromosomes were used for autokaryotyping, whereas red and green images from competitive hybridization staining provided the signals for deriving the intensity ratio profile between normal and tumor samples. Genomic aberrations such as gains (amplification) or losses (deletion) at certain chromosome regions were defined when the mean green (from tumor part) to red (from normal part) ratios is above 1.2 or below 0.8 , respectively.

\section{Statistical Analysis}

We adopted two-tailed Fisher's exact test to assess the correlation of genetic aberrances with clinicopathologic features. The incidence of 11q13 loss was compared with the accumulated data from prior CGH studies on foregut carcinoids by two-tailed Fisher's exact test. We made the comparison because prior CGH studies of foregut carcinoids $^{10-12}$ disclosed a high incidence of allelic losses at this locus where the MEN1 gene is located. The computation was achieved using the Stata program (Stata Corp., TX, USA, 1997).

\section{Results}

The patients ranged in age from 32 to 74 years (mean: 53.8 years). There was a male predominance $(\mathrm{M}: \mathrm{F}=10: 1)$ partly owing to the patient pattern at the institute, where male patients predominate. One patient (No. 8) had a recurrence 2 years after the primary tumor. Both the primary and recurrent tumors were submitted for $\mathrm{CGH}$ analyses. One patient (No. 7) had a synchronous pancreatic neuroendocrine tumor, adrenocortical hyperplasia and parathyroid hyperplasia, suggesting MEN1. The patient also had familial history of MEN1. The pancreatic neuroendocrine tumor was assessed for CGH in parallel. None of the patients had Cushing's syndrome or other paraneoplastic syndromes.

Most of cases were either minimally invasive (stage II) or widely invasive (stage III). Histologically, three of 11 cases were classified as low grade, eight as intermediate grade and one as high grade.

Follow-up data could be obtained for all of the patients. Five patients died of the disease (survival times: 11-52 months; mean: 31.8 months). The other patients were alive without evidence of disease during the follow-up duration (follow-up times: 20-158 months; mean: 91.7 months).

\section{CGH}

Genomic alterations were detected in nine cases. Gains were observed in chromosome regions X (four cases), 8 (three cases), 18 (two cases) and 20p (one case). Losses were observed in chromosomes 6 (two cases), 13q (two cases), 3 p (one case), 9q (one case) and 11q (one case) (Figures 2 and 3). The genomic aberrations of the recurrent tumor of case no. 8 were nearly identical to those of the primary tumor excepting the addition of a gain on the $\mathrm{X}$ chromosome in the recurrent tumor. The chromosomal abnormalities detected in the thymic and pancreatic neuroendocrine tumors of the patient with MEN1 (case no. 7) were entirely different. The clinicopathologic features and CGH findings are tabulated in Table 1.

\section{Statistical Analysis}

No statistical significance could be found between the CGH findings and clinicopathologic features including stage, grade and survival $(P>0.05)$. A meta-analysis of prior CGH studies ${ }^{10-12}$ on foregut carcinoids showed 25 of 69 (36\%) cases as having an 11 q13 loss. On the contrary, none of our cases showed deletion at 11q13. The difference regarding 
the incidences of an 11q13 loss was statistically significant between foregut and thymic neuroendocrine tumors $(P=0.0144)$.

\section{Discussion}

CGH is an efficient tool to provide a profile of the entire tumor genome, though it may not be sensitive enough to discern small chromosomal instability. It can be applied to archival formalin-fixed, paraffinembedded tissue; thus it makes possible a cytogenetic examination for rare diseases, when fresh

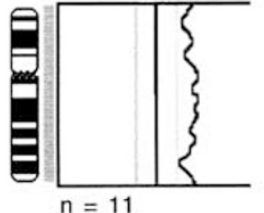

$x$

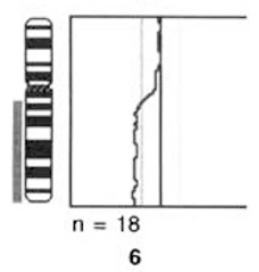

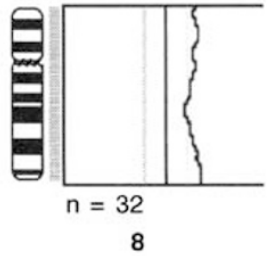
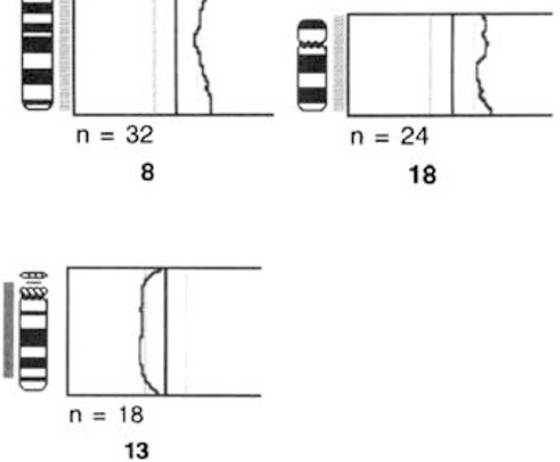

Figure 2 Representative examples of genomic imbalances on chromosomes X, 8, 18, 6 and 13 detected by CGH. The chromosomal ideograms are shown along with the mean ratio profiles. $N$ indicates number of metaphases analyzed. Lower/ upper thresholds are the ratio values of 0.8 and 1.2. Gains of chromosomal material are shown as a bar on the right side of the ideogram; losses are depicted along the left side. tissue is hardly attainable. Using the same protocol, we have successfully performed CGH on the paraffin specimens of other kinds of cancer. ${ }^{9,13}$ In this study, we delineated the genetic alterations of thymic neuroendocrine tumor-a rare and hitherto poorly characterized neoplasm. To our knowledge, our study is the first CGH analysis on thymic neuroendocrine tumors.

Two limitations intrinsic to this study should be addressed. First, due to the rarity of the neoplasm, the case numbers were low. The limited cases did not allow a statistically significant statement with regard to the genetic differences among histologic subtypes and clinical stages. The low number of cases analyzed in the present study also hampered drawing conclusions on genetic alterations in these tumors. Second, even though we selected samples in which the tumor cells accounted for more than $80 \%$ of cell population, there was still a small population of normal cells that might confound the results. Despite the limitations, we still have successfully detected a few gross chromosomal imbalances in nine of 11 cases. Nevertheless, the few genetic alterations are well in line with the recently published data of Goto et $a l^{14}$ on thymic neuroendocrine tumors showing diploidy in most of the cases they studied.

We did not find any deletion at the locus 11q13 where the MEN1 gene is located, and we only detected deletion on 11q24-25 in one case (case 6). Contrarily, prior CGH and LOH studies disclosed a high incidence of allelic losses on $11 \mathrm{q}$ in both sporadic and MEN1-associated neuroendocrine tumors of the bronchopulmonary ${ }^{10-12,15-18}$ and the digestive systems. ${ }^{19-23}$ The rate of $11 \mathrm{q} 13 \mathrm{LOH}$ was

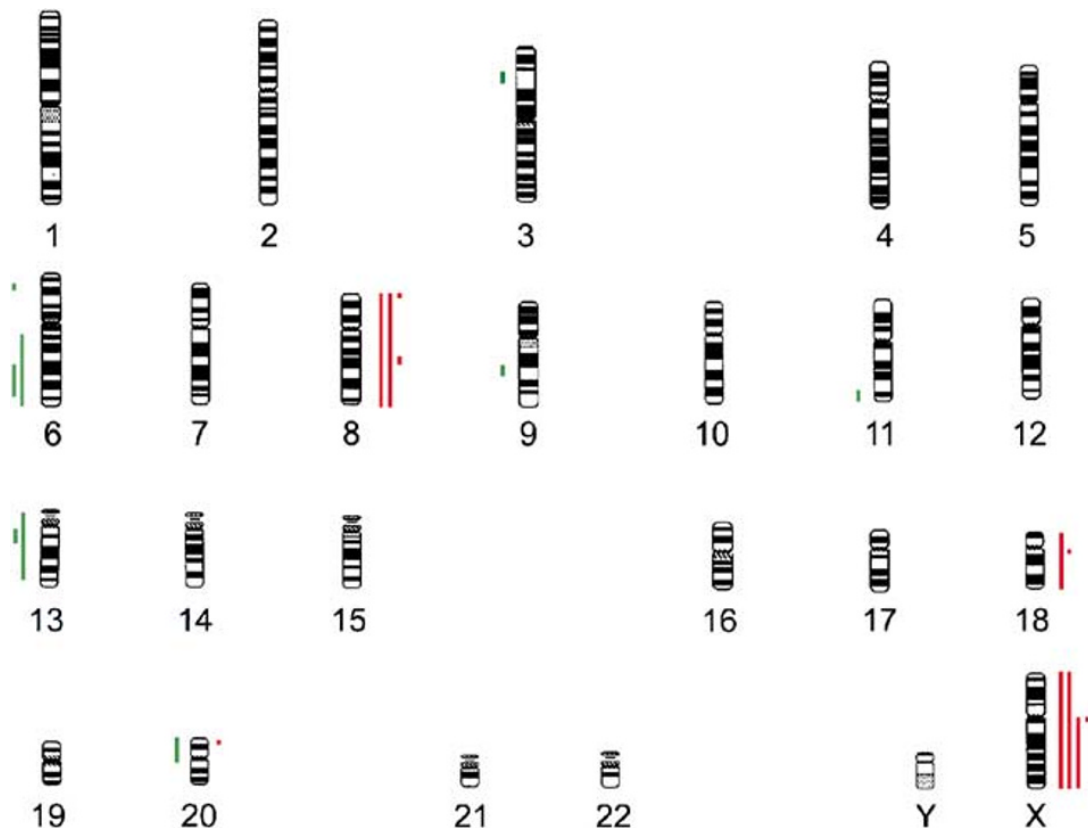

Figure 3 Compilation of CGH analysis of the chromosomal aberrations in nine thymic neuroendocrine (carcinoid) tumors. Vertical lines on the right side of each chromosome ideogram represent gains. Vertical lines on the left side represent losses. 
Table 1 Clinicopathologic features and CGH findings

\begin{tabular}{|c|c|c|c|c|c|c|c|c|}
\hline No. & Gender & Age (years) & Stage & Grade & Status & Survival (months) & Chromosomal gain & Chromosomal loss \\
\hline 1 & M & 65 & 2 & Low & DOD & 52 & $\mathrm{Nil}$ & $\mathrm{Nil}$ \\
\hline 2 & $\mathrm{M}$ & 32 & 1 & Low & AW & 107 & $8 p, 8 q$ & Nil \\
\hline 3 & $\mathrm{M}$ & 51 & 2 & Low & AW & 156 & $X^{1}$ & Nil \\
\hline 4 & $\mathrm{M}$ & 71 & 2 & Intermediate & DOD & 35 & $18 p, 18 q$ & $13 q 12-14$ \\
\hline 5 & $\mathrm{M}$ & 74 & 2 & Intermediate & DOD & 11 & $18 q 11-12.1$ & $\mathrm{Nil}$ \\
\hline 6 & $\mathrm{M}$ & 39 & 3 & Intermediate & DOD & 24 & $8 p, 8 q$ & $3 p 21,9 q 21-22,11 q 24-25$ \\
\hline 7 & $\mathrm{M}$ & 42 & 2 & Intermediate & DOD & 40 & 8p23, 8q21.1-23, Xq & Nil \\
\hline $7 p$ & - & - & - & - & - & - & Nil & 16p12-13.1 \\
\hline 8 & $\mathrm{M}$ & 52 & 3 & Intermediate & AW & 84 & Nil & 6q13-27, 20p, 20q11 \\
\hline $8 \mathrm{r}$ & - & - & 1 & Intermediate & - & - & $\mathrm{Xq11-13}$ & $6 q 13-27,20 p, 20 q 11$ \\
\hline 9 & $\mathrm{~F}$ & 60 & 2 & Intermediate & AW & 25 & Nil & Nil \\
\hline 10 & $\mathrm{M}$ & 41 & 3 & Intermediate & AW & 20 & $\mathrm{X}$ & Nil \\
\hline 11 & $\mathrm{M}$ & 65 & 3 & High & AW & 158 & 20p12-13 & $6 \mathrm{p} 22-23,6 q 22-25,13 q 11-33$ \\
\hline
\end{tabular}

DOD: died of disease; AW: alive and well; 7p: pancreatic neuroendocrine tumor of no. 7; 8r: recurrent thymic neuroendocrine tumor of no. 8 .

reported as high as $78 \%$ in foregut carcinoid tumors. ${ }^{17}$ Our CGH data are in agreement with the findings in previously reported LOH studies on 12 MEN1-associated thymic neuroendocrine tumors, all of which demonstrated retention of heterozygosity at the locus $11 \mathrm{q} 13 .{ }^{6-8}$ The absence of $11 \mathrm{q}$ alterations in thymic neuroendocrine tumors supports the distinction from their presumed foregut counterparts at the cytogenetic level despite overlapping histologic features.

On the other hand, we observed a few cytogenetic aberrations not usually present in the foregut neuroendocrine tumors. Two cases had variable lengths of genetic losses on chromosome 6 . Interestingly, chromosome 6 has been reported to be a frequent target of deletion in thymoma and thymic carcinoma. ${ }^{24-26}$ Two cases had $13 q$ loss. Allelic loss on $13 q$ have been observed in thymic epithelial tumors ${ }^{25,26}$ and non-small-cell lung carcinomas. ${ }^{27,28}$ The tumor suppressor genes mapped to the 13q1214 regions include the retinoblastoma gene and the hereditary breast cancer susceptibility gene (BRCA2). To date, there have been no studies addressing those genes in thymic neuroendocrine tumors. We consider these findings as having certain histogenetic implications, but it requires recruiting more cases for further investigation.

Regarding the chromosomal gains, four cases had gain on $\mathrm{X}$ chromosome and three cases had gain on chromosome 8 . The amplification spanned over the whole chromosome in some of cases. We suspect a trisomy $\mathrm{X}$ or 8 in those cases. Karyotyping or fluorescent in situ hybridization would be helpful to validate this speculation. Gain of the entire or partial copy of X chromosome have been found at high frequency in endocrine tumors such as pituitary adenoma ${ }^{29}$ and adrenocortical adenoma, ${ }^{30}$ as well as several solid and hematopoietic malignancies. $^{31-33}$ There is growing evidence that a few Xlinked genes are involved in the development of neoplasia. ${ }^{34}$ Trisomy 8 frequently occurs in myelocytic malignancies ${ }^{35}$ and breast cancers. ${ }^{36,37}$ Multiplication of $8 \mathrm{q}$ has also been described in cancers of the breast and the liver. ${ }^{38,39}$ The candidate oncogene on $8 \mathrm{q}$ includes c-myc, which has yet to be investigated in thymic neuroendocrine tumors.

Finally, one case exhibited complete amplification of the chromosome 18, and one case showed partial gain on the long arm. Gain of 18q has been reported in neuroendocrine carcinomas of lung and it is presumed to correlate to the expression of oncogene BCL2 that is located at $18 \mathrm{q} 21 .^{12,28}$ Supplementing that hypothesis, a recent study also demonstrated overexpression of BCL2 protein in a portion of thymic neuroendocrine tumors. ${ }^{40}$

In conclusion, we identified chromosomal abnormalities chiefly involving chromosome 6, 8, 13, 18 and $\mathrm{X}$ in thymic neuroendocrine tumors. Based on our findings, in conjunction with previous ones, we favor a fundamental molecular divergence between thymic neuroendocrine tumors and foregut carcinoid tumors. Owing to the limited sample size, we are unable to obtain statistical significances concerning the correlation between cytogenetic changes and clinicopathologic parameters such as stage, grade and survival; however, our data could still offer new insights into the underlying cytogenetic mechanism of thymic neuroendocrine tumors and open an avenue for further researches regarding this peculiar neoplasm.

\section{Acknowledgements}

This work was supported financially by Grant No. V92-304 from the Taipei Veterans General Hospital and Grant No. NSC 91-2314-B-010-072 from the Taiwan National Science Council. We thank Gerald W Kamp for editing the manuscript.

\section{References}

1 Rosai J, Higa E. Mediastinal endocrine neoplasm, of probable thymic origin, related to carcinoid tumor. 
Clinicopathologic study of 8 cases. Cancer 1972;29: 1061-1074.

2 Chaer R, Massad MG, Evans A, et al. Primary neuroendocrine tumors of the thymus. Ann Thorac Surg 2002;74:1733-1740.

3 Moran CA, Suster S. Neuroendocrine carcinomas (carcinoid tumor) of the thymus. A clinicopathologic analysis of 80 cases. Am J Clin Pathol 2000;114: 100-110.

4 Gal AA, Kornstein MJ, Cohen C, et al. Neuroendocrine tumors of the thymus: a clinicopathological and prognostic study. Ann Thorac Surg 2001;72:1179-1182.

5 Rosai J. Histological Typing of Tumours of the Thymus 2nd edn. Springer-Verlag: New York: 1999.

6 Gibril F, Chen YJ, Schrump DS, et al. Prospective study of thymic carcinoids in patients with multiple endocrine neoplasia type 1. J Clin Endocrinol Metab 2003; 88:1066-1081.

7 Hessman O, Skogseid B, Westin G, et al. Multiple allelic deletions and intratumoral genetic heterogeneity in MEN1 pancreatic tumors. J Clin Endocrinol Metab 2001;86:1355-1361.

8 Teh BT, Zedenius J, Kytola S, et al. Thymic carcinoids in multiple endocrine neoplasia type 1. Ann Surg 1998;228:99-105.

9 Yen CC, Chen YJ, Chen JT, et al. Comparative genomic hybridization of esophageal squamous cell carcinoma: correlations between chromosomal aberrations and disease progression/prognosis. Cancer 2001;92: 2769-2777.

10 Ullmann R, Petzmann S, Klemen H, et al. The position of pulmonary carcinoids within the spectrum of neuroendocrine tumors of the lung and other tissues. Genes Chromosomes Cancer 2002;34:78-85.

11 Zhao J, de Krijger RR, Meier D, et al. Genomic alterations in well-differentiated gastrointestinal and bronchial neuroendocrine tumors (carcinoids): marked differences indicating diversity in molecular pathogenesis. Am J Pathol 2000;157:1431-1438.

12 Walch AK, Zitzelsberger HF, Aubele MM, et al. Typical and atypical carcinoid tumors of the lung are characterized by 11q deletions as detected by comparative genomic hybridization. Am J Pathol 1998;153:10891098.

13 Chen YJ, Yeh SH, Chen JT, et al. Chromosomal changes and clonality relationship between primary and recurrent hepatocellular carcinoma. Gastroenterology 2000;119:431-440.

14 Goto K, Kodama T, Matsuno Y, et al. Clinicopathologic and DNA cytometric analysis of carcinoid tumors of the thymus. Mod Pathol 2001;14:985-994.

15 Debelenko LV, Swalwell JI, Kelley MJ, et al. MEN1 gene mutation analysis of high-grade neuroendocrine lung carcinoma. Genes Chromosomes Cancer 2000;28: 58-65.

16 Petzmann S, Ullmann $\mathrm{R}$, Klemen $\mathrm{H}$, et al. Loss of heterozygosity on chromosome arm 11q in lung carcinoids. Hum Pathol 2001;32:333-338.

17 Jakobovitz O, Nass D, DeMarco L, et al. Carcinoid tumors frequently display genetic abnormalities involving chromosome 11. J Clin Endocrinol Metab 1996;81: 3164-3167.

18 Onuki N, Wistuba II, Travis WD, et al. Genetic changes in the spectrum of neuroendocrine lung tumors. Cancer 1999;85:600-607.

19 Bale AE, Norton JA, Wong EL, et al. Allelic loss on chromosome 11 in hereditary and sporadic tumors related to familial multiple endocrine neoplasia type 1. Cancer Res 1991;51:1154-1157.

20 D'Adda T, Keller G, Bordi C, et al. Loss of heterozygosity in 11q13-14 regions in gastric neuroendocrine tumors not associated with multiple endocrine neoplasia type 1 syndrome. Lab Invest 1999;79:671-677.

21 Debelenko LV, Emmert-Buck MR, Zhuang Z, et al. The multiple endocrine neoplasia type I gene locus is involved in the pathogenesis of type II gastric carcinoids. Gastroenterology 1997;113:773-781.

22 Speel EJ, Richter J, Moch H, et al. Genetic differences in endocrine pancreatic tumor subtypes detected by comparative genomic hybridization. Am J Pathol 1999;155:1787-1794.

23 Stumpf E, Aalto Y, Hoog A, et al. Chromosomal alterations in human pancreatic endocrine tumors. Genes Chromosomes Cancer 2000;29:83-87.

24 Inoue M, Marx A, Zettl A, et al. Chromosome 6 suffers frequent and multiple aberrations in thymoma. Am J Pathol 2002;161:1507-1513.

25 Inoue M, Starostik P, Zettl A, et al. Correlating genetic aberrations with World Health Organization-defined histology and stage across the spectrum of thymomas. Cancer Res 2003;63:3708-3715.

26 Zhou R, Zettl A, Strobel P, et al. Thymic epithelial tumors can develop along two different pathogenetic pathways. Am J Pathol 2001;159:1853-1860.

27 Kwong FM, Wong PS, Lung ML. Genetic alterations detected on chromosomes 13 and 14 in Chinese nonsmall cell lung carcinomas. Cancer Lett 2003;192: 189-198.

28 Michelland S, Gazzeri S, Brambilla E, et al. Comparison of chromosomal imbalances in neuroendocrine and non-small-cell lung carcinomas. Cancer Genet Cytogenet 1999;114:22-30.

29 Trautmann K, Thakker RV, Ellison DW, et al. Chromosomal aberrations in sporadic pituitary tumors. Int J Cancer 2001;91:809-814.

30 Russell AJ, Sibbald J, Haak $\mathrm{H}$, et al. Increasing genome instability in adrenocortical carcinoma progression with involvement of chromosomes 3,9 and $\mathrm{X}$ at the adenoma stage. Br J Cancer 1999;81: $684-689$

$31 \mathrm{Hu}$ J, Khanna V, Jones MM, et al. Genomic imbalances in ovarian borderline serous and mucinous tumors. Cancer Genet Cytogenet 2002;139:18-23.

32 Renedo M, Martinez-Delgado B, Arranz E, et al. Chromosomal changes pattern and gene amplification in T cell non-Hodgkin's lymphomas. Leukemia 2001; 15:1627-1632.

33 Terracciano LM, Bernasconi B, Ruck P, et al. Comparative genomic hybridization analysis of hepatoblastoma reveals high frequency of X-chromosome gains and similarities between epithelial and stromal components. Hum Pathol 2003;34:864-871.

34 Brown CJ. Role of the X chromosome in cancer. J Natl Cancer Inst 1996;88:480-482.

35 Paulsson K, Fioretos T, Strombeck B, et al. Trisomy 8 as the sole chromosomal aberration in myelocytic malignancies: a multicolor and locus-specific fluorescence in situ hybridization study. Cancer Genet Cytogenet 2003;140:66-69.

36 Afify A, Bland KI, Mark HF. Fluorescent in situ hybridization assessment of chromosome 8 copy number in breast cancer. Breast Cancer Res Treat 1996;38:201-208. 
37 Bullerdiek J, Leuschner E, Taquia E, et al. Trisomy 8 as a recurrent clonal abnormality in breast cancer? Cancer Genet Cytogenet 1993;65:64-67.

38 Fujiwara Y, Monden M, Mori T, et al. Frequent multiplication of the long arm of chromosome 8 in hepatocellular carcinoma. Cancer Res 1993;53: 857-860.
39 Yokota T, Yoshimoto M, Akiyama F, et al. Frequent multiplication of chromosomal region 8q24.1 associated with aggressive histologic types of breast cancers. Cancer Lett 1999;139:7-13.

40 Gal AA, Sheppard MN, Nolen JD, et al. p53, cellular proliferation, and apoptosis-related factors in thymic neuroendocrine tumors. Mod Pathol 2004;17:33-39. 